\title{
QFT Derivation of the Decay Law of an Unstable Particle with Nonzero Momentum
}

\author{
Francesco Giacosa ${ }^{1,2}$ \\ ${ }^{1}$ Institute of Physics, Jan-Kochanowski University, Ul. Swietokrzyska 15, 25-406 Kielce, Poland \\ ${ }^{2}$ Institute for Theoretical Physics, J. W. Goethe University, Max-von-Laue-Str. 1, 60438 Frankfurt, Germany \\ Correspondence should be addressed to Francesco Giacosa; fgiacosa@ujk.edu.pl
}

Received 7 April 2018; Accepted 3 June 2018; Published 26 June 2018

Academic Editor: Neelima G. Kelkar

Copyright (C) 2018 Francesco Giacosa. This is an open access article distributed under the Creative Commons Attribution License, which permits unrestricted use, distribution, and reproduction in any medium, provided the original work is properly cited. The publication of this article was funded by SCOAP $^{3}$.

\begin{abstract}
We present a quantum field theoretical derivation of the nondecay probability of an unstable particle with nonzero threemomentum $\mathbf{p}$. To this end, we use the (fully resummed) propagator of the unstable particle, denoted as $S$, to obtain the energy probability distribution, called $d_{S}^{\mathbf{p}}(E)$, as the imaginary part of the propagator. The nondecay probability amplitude of the particle $S$ with momentum $\mathbf{p}$ turns out to be, as usual, its Fourier transform: $a_{S}^{\mathbf{p}}(t)=\int_{\sqrt{m_{t h}^{2}+\mathbf{p}^{2}}}^{\infty} d E d_{S}^{\mathbf{p}}(E) e^{-i E t}$ ( $m_{t h}$ is the lowest energy threshold in the rest frame of $S$ and corresponds to the sum of masses of the decay products). Upon a variable transformation, one can rewrite it as $a_{S}^{\mathbf{p}}(t)=\int_{m_{t h}}^{\infty} d m d_{S}^{0}(m) e^{-i \sqrt{m_{t h}^{2}+\mathbf{p}^{2}}}$ [here, $d_{S}^{0}(m) \equiv d_{S}(m)$ is the usual spectral function (or mass distribution) in the rest frame]. Hence, the latter expression, previously obtained by different approaches, is here confirmed in an independent and, most importantly, covariant QFT-based approach. Its consequences are not yet fully explored but appear to be quite surprising (such as the fact that the usual time-dilatation formula does not apply); thus its firm understanding and investigation can be a fruitful subject of future research.
\end{abstract}

\section{Introduction}

The study of the decay law is a fundamental part of quantum mechanics (QM). It is now theoretically [1-9] and experimentally $[10,11]$ established that deviations from the exponential decay exist, but they are usually small. Such deviations are also present in quantum field theory (QFT) $[9,12]$.

An interesting question addresses the decay of an unstable particle with nonzero momentum p. In [13-17], it was shown-by using QM-based approaches enlarged to include special relativity - that the nondecay probability of an unstable particle $S$ with momentum $\mathbf{p}$ is given by (in natural units)

$$
\begin{aligned}
& P_{S}^{\mathbf{p}}(t)=\left|a_{S}^{\mathbf{p}}(t)\right|^{2} \\
& \text { with } a_{S}^{\mathbf{p}}(t)=\int_{m_{t h}}^{\infty} \mathrm{dm} d_{S}(m) e^{-i \sqrt{m^{2}+\mathbf{p}^{2}} t},
\end{aligned}
$$

where $d_{S}(m)$ is the energy (or mass) distribution of $S$ in its rest frame $\left[\mathrm{dm} d_{S}(m)\right.$ is the probability that the unstable state $S$ has energy (or mass) between $m$ and $m+\mathrm{dm}]$. A review of the derivation is presented in Section 2. Quite remarkably, there are many interesting properties linked to this equation, which include deviations from the standard dilatation formula; see below.

The purpose of this work is straightforward: we derive (1) in a QFT framework (see Section 3). We thus confirm its validity and, as a consequence, its peculiar features. For definiteness, an underlying Lagrangian involving scalar fields is introduced, but our discussion is valid for any spin of the unstable particle and of the decay products. A central quantity of our study is the relativistic propagator of the unstable particle $S$ : the mass distribution $d_{S}(m)$ is then obtained by the imaginary part of the propagator.

In this Introduction, we recall some basic and striking features connected to (1). The normalization

$$
\int_{m_{t h}}^{\infty} \mathrm{dm} d_{S}(m)=1
$$


is a crucial feature of the spectral function, implying that $a_{S}^{\mathbf{p}}(0)=1$. It must be valid both in QM and in QFT. Here, without loss of generality, we set the lower limit of the integral to $m_{t h} \geq 0$. In fact, a minimal energy $m_{t h}$ is present in each physical system; in particular, for a (relevant for us) relativistic system, it is given by the sum of the rest masses of the produced particles $\left(m_{t h}=m_{1}+m_{2}+\cdots \geq 0\right)$. Clearly, for $\mathbf{p}=\mathbf{0}$, (1) reduces to the usual expression

$$
P_{S}^{r e s t}(t)=P_{S}^{0}(t)=\left|a_{S}^{0}(t)\right|^{2}=\left|\int_{m_{t h}}^{\infty} \operatorname{dm} d_{S}(m) e^{-i m t}\right|^{2} .
$$

A detailed study of (1) shows that the usual time dilatation does not hold:

$$
P_{S}^{\mathbf{p}}(t) \neq P_{S}^{\text {rest }}\left(t \frac{M}{\sqrt{M^{2}+\mathbf{p}^{2}}}\right),
$$

where $M$ is the mass of the state $S$ defined, for instance, as the position of the peak of the distribution $d_{S}(m)$; in general, however, other definitions are possible, such as the real part of the pole of the propagator; see Section 3. The point is that, no matter which definition one takes, expression (4) remains an inequality.

It is always instructive to investigate the exponential limit, in which the spectral function of the state $S$ reads $[18,19]$

$$
d_{S}^{B W}(m)=\frac{\Gamma}{2 \pi}\left[(m-M)^{2}+\frac{\Gamma^{2}}{4}\right]^{-1},
$$

where $M$ is the "mass of the unstable state" corresponding to the peak. Even if the spectral function $d_{S}^{B W}(m)$ is clearly unphysical because there is no minimal energy $\left(m_{t h} \rightarrow-\infty\right)$, in many physical cases it is a good approximation for a quite broad energy range. Here, the decay amplitude and the decay law in the rest frame of the decaying particle notoriously read

$$
\begin{aligned}
a_{S}^{B W, \mathbf{0}}(t) & =e^{-i M t-\Gamma t / 2} \longrightarrow \\
P_{S}^{B W, \text { rest }}(t) & =e^{-\Gamma t} .
\end{aligned}
$$

When a nonzero momentum is considered, the nondecay probability is still an exponential given by

$$
P_{S}^{B W, \mathbf{p}}(t)=e^{-\Gamma_{\mathbf{p}} t}
$$

where the width is [17]

$$
\begin{aligned}
& \Gamma_{\mathbf{p}} \\
& =\sqrt{2} \sqrt{\left[\left(M^{2}-\frac{\Gamma^{2}}{4}+\mathbf{p}^{2}\right)^{2}+M^{2} \Gamma^{2}\right]^{1 / 2}-\left(M^{2}-\frac{\Gamma^{2}}{4}+\mathbf{p}^{2}\right)} .
\end{aligned}
$$

Clearly, $\Gamma_{\mathbf{p}=\mathbf{0}}=\Gamma$. One realizes, however, that $\Gamma_{\mathbf{p}}$ differs from the naively expected standard time-dilatation formula, according to which the decay width of an unstable state with momentum $\mathbf{p}$ should simply be

$$
\frac{\Gamma M}{\sqrt{\mathbf{p}^{2}+M^{2}}}=\gamma \Gamma \text {. }
$$

Namely, the quantity $\gamma=\sqrt{\mathbf{p}^{2}+M^{2}} / M=1 / \sqrt{1-\mathbf{v}^{2}}$ is the usual dilatation factor for a state with (definite) energy $M$. Deviations between (8) and (9) are very small, as the numerical discussion in [17] shows. Although not measurable by current experiments [20], the very fact that deviations exist is very interesting and deserves further study.

As a last point, it should be stressed that in this work we consider unstable states with a definite momentum p. This is a subtle point: while for a state with definite energy, a boost and momentum translation are equivalent, this is not so for an unstable state, since it is not an energy eigenstate. Even more surprisingly, a boost of an unstable state is a quantum state whose nondecay probability is actually zero: it is already decayed (on the contrary, its survival probability presents a peculiar time contraction [21]). In other words, a boosted muon consists of an electron and two neutrinos $[15,17]$. In this sense, the boost mixes the Hilbert subspace of the undecayed states with the subspace of the decay products; see [17] for details. There, it is also discussed why the basis of unstable states contains states with definite three-momentum. Indeed, the investigation of this paper also confirms this aspect: unstable states with definite momentum naturally follow from the study of its propagator in QFT.

The paper is organized in this way: in Section 2 we recall the QM derivation of (1), while in Section 3-the key part of this paper-we present this derivation in a QFT context. In the end, in Section 4 we describe our conclusions.

\section{Recall of the QM-Based Derivation of (1)}

For completeness, we report here the "standard" derivation of (1). To this end, we use the arguments presented in [17], but similar ones can be found in [13-16].

We consider a system described by the Hamiltonian $H$, whose eigenstates are denoted as

$$
|m, \mathbf{p}\rangle=U_{\mathbf{p}}|m, \mathbf{0}\rangle,
$$

where $U_{\mathbf{p}}$ is the unitary operator associated with the translation in momentum space. Standard normalization expressions are assumed:

$$
\left\langle m_{1}, \mathbf{p}_{1} \mid m_{2}, \mathbf{p}_{2}\right\rangle=\delta\left(m_{1}-m_{2}\right) \delta\left(\mathbf{p}_{1}-\mathbf{p}_{2}\right) .
$$

The state $|m, \mathbf{p}\rangle$ has definite energy,

$$
H|m, \mathbf{p}\rangle=\sqrt{\mathbf{p}^{2}+m^{2}}|m, \mathbf{p}\rangle,
$$

definite momentum,

$$
\mathbf{P}|m, \mathbf{p}\rangle=\mathbf{p}|m, \mathbf{p}\rangle,
$$

and definite velocity $\mathbf{p} / \sqrt{\mathbf{p}^{2}+m^{2}}$. Note, assuming that the energy of $|m, \mathbf{p}\rangle$ is $\sqrt{\mathbf{p}^{2}+m^{2}}$, we have a relativistic spectrum.

Formally, the Hamiltonian can be written as

$$
\begin{aligned}
H & =\int \mathrm{d}^{3} \mathrm{p} \int_{m_{t h}}^{\infty} d m \sqrt{\mathbf{p}^{2}+m^{2}}|m, \mathbf{p}\rangle\langle m, \mathbf{p}| \\
& =\int \mathrm{d}^{3} \mathrm{p} H_{\mathbf{p}}
\end{aligned}
$$


where

$$
H_{\mathbf{p}}=\int_{m_{t h}}^{\infty} d m \sqrt{\mathbf{p}^{2}+m^{2}}|m, \mathbf{p}\rangle\langle m, \mathbf{p}|
$$

is the effective Hamiltonian in the subspace of states with definite momentum $\mathbf{p}$.

Let us now consider an unstable state $S$ in its rest frame. The corresponding quantum state at rest is assumed to be

$$
|S, \mathbf{0}\rangle=\int_{m_{t h}}^{\infty} \mathrm{dm} \alpha_{S}(m)|m, \mathbf{0}\rangle,
$$

where $\alpha_{S}(m)$ is the probability amplitude that the state $S$ has energy $m$. Hence, it is natural that the quantity $d_{S}(m)=$ $\left|\alpha_{S}(m)\right|^{2}$ is the mass distribution: $d_{S}(m) \mathrm{dm}$ is the probability that the unstable particle $S$ has a mass between $m$ and $m+\mathrm{dm}$. As a consequence, $\int_{0}^{\infty} \mathrm{dm} d_{S}(m)=1$, as already discussed in the Introduction.

For the states of zero momentum, the Hamiltonian $H_{\mathrm{p}=\mathbf{0}}$ can be expressed in terms of the undecayed state $|S, \mathbf{0}\rangle$ and its decay products in the form of a Lee Hamiltonian [22] (similar effective Hamiltonians are used also in quantum mechanics $[6,19]$ and quantum field theory $[9,23])$ :

$$
\begin{aligned}
H_{\mathbf{p}=\mathbf{0}} & =\int_{m_{\text {th }}}^{\infty} \mathrm{dm} m|m, \mathbf{0}\rangle\langle m, \mathbf{0}| \\
= & M_{0}|S, \mathbf{0}\rangle\left\langle S, \mathbf{0}\left|+\int \mathrm{d}^{3} \mathrm{k} \omega(\mathbf{k})\right| \mathbf{k}, \mathbf{0}\right\rangle\langle\mathbf{k}, \mathbf{0}| \\
& +\int \frac{\mathrm{d}^{3} \mathrm{k}}{(2 \pi)^{3 / 2}} g f(\mathbf{k})[|S, \mathbf{0}\rangle\langle\mathbf{k}, \mathbf{0}|+| \mathbf{k}, \mathbf{0}\rangle\langle S, \mathbf{0}|],
\end{aligned}
$$

where $|\mathbf{k}, \mathbf{0}\rangle$ represents a decay product with vanishing total momentum: in the two-body decay case, $|\mathbf{k}, \mathbf{0}\rangle$ describes two particles, the first with momentum $\mathbf{k}$ and the second with momentum $-\mathbf{k}$, hence

$$
\omega(\mathbf{k})=\sqrt{\mathbf{k}^{2}+m_{1}^{2}}+\sqrt{\mathbf{k}^{2}+m_{2}^{2}} .
$$

The last term in (17) represents the "mixing" between $|S, \mathbf{0}\rangle$ and $|\mathbf{k}, \mathbf{0}\rangle$, which causes the decay of the former into the latter. Moreover, $g$ is a coupling constant and $f(\mathbf{k})$ encodes the dependence of the mixing on the momentum of the produced particles. The explicit expressions connecting the states $|\mathbf{k}, \mathbf{0}\rangle$ to $|m, \mathbf{0}\rangle$ formally read

$$
|\mathbf{k}, \mathbf{0}\rangle=\int_{m_{t h}}^{\infty} \mathrm{dm} \beta_{\mathbf{k}}(m)|m, \mathbf{0}\rangle
$$

where $\beta_{\mathbf{k}}(m)$ can be found by diagonalizing the Hamiltonian (17).

Let us now consider an unstable state with definite momentum $\mathbf{p}$, which is denoted as $|S, \mathbf{p}\rangle$ :

$$
|S, \mathbf{p}\rangle=U_{\mathbf{p}}|S, \mathbf{0}\rangle=\int_{m_{t h}}^{\infty} \mathrm{dm} \alpha_{S}(m)|m, \mathbf{p}\rangle .
$$

The normalization

$$
\left\langle S, \mathbf{p}_{1} \mid S, \mathbf{p}_{2}\right\rangle=\delta\left(\mathbf{p}_{1}-\mathbf{p}_{2}\right)
$$

follows. Note that (20) is not a state with definite velocity. This is due to the fact that each state $|m, \mathbf{p}\rangle$ in the superposition has a different velocity $\mathbf{p} / \sqrt{\mathbf{p}^{2}+m^{2}}$. The subset of Hilbert space given by $\left\{|S, \mathbf{p}\rangle \forall \mathbf{p} \subset R^{2}\right\}$ represents the set of all undecayed quantum states of the system under study.

The form of the Hamiltonian $H_{\mathbf{p}}$ in terms of the states $|S, \mathbf{p}\rangle$ and $U_{\mathbf{p}}|\mathbf{k}, \mathbf{0}\rangle=|\mathbf{k}, \mathbf{p}\rangle$ can be in principle derived by using the expressions above. Together with (20), one shall also take (19) and apply $U_{\mathbf{p}}$ in order to get

$$
U_{\mathbf{p}}|\mathbf{k}, \mathbf{0}\rangle=|\mathbf{k}, \mathbf{p}\rangle=\int_{m_{t h}}^{\infty} \mathrm{dm} \beta_{\mathbf{k}}(m)|m, \mathbf{p}\rangle .
$$

Then, one should invert (20) and (22) and insert it into $H_{\mathbf{p}}$ of (15). However, its explicit expression is definitely not trivial but, fortunately, also not needed in the present work. Hence, we do not attempt to write it down here.

We now turn to the nondecay amplitude of the state $S$. In its rest frame $(\mathbf{p}=\mathbf{0})$, upon starting from a properly normalized state with zero momentum, $|S, \mathbf{0}\rangle / \sqrt{\delta(\mathbf{0})}$, one obtains the usual expression

$$
\begin{aligned}
a_{S}^{\mathbf{0}}(t) & =\frac{1}{\delta(\mathbf{0})}\left\langle S, \mathbf{0}\left|e^{-i H t}\right| S, \mathbf{0}\right\rangle \\
& =\frac{1}{\delta(\mathbf{0})} \int_{m_{t h}}^{\infty} \mathrm{dm}_{1} \mathrm{dm}_{2}\left\langle m_{1}, \mathbf{0}\left|e^{-i H t}\right| m_{2}, \mathbf{0}\right\rangle \\
& =\int_{m_{t h}}^{\infty} \mathrm{dm} d_{S}(m) e^{-i m t},
\end{aligned}
$$

in agreement with (3). The theory of decay is discussed in great detail for the case $\mathbf{p}=\mathbf{0}$ in $[4,6,7,9]$ and references therein. Note here the nondecay probability coincides with the survival probability (that is, the probability that the state did not change), but in general this is not the case [17].

Next, we consider a normalized unstable state $S$ with nonzero momentum: $|S, \mathbf{p}\rangle / \sqrt{\delta(\mathbf{0})}$. The resulting nondecay probability amplitude

$$
\begin{aligned}
a_{S}^{\mathbf{p}}(t) & =\frac{1}{\delta(\mathbf{0})}\left\langle S, \mathbf{p}\left|e^{-i H t}\right| S, \mathbf{p}\right\rangle \\
& =\frac{1}{\delta(\mathbf{0})} \int_{m_{t h}}^{\infty} \mathrm{dm}_{1} \mathrm{dm}_{2}\left\langle m_{1}, \mathbf{p}\left|e^{-i H t}\right| m_{2}, \mathbf{p}\right\rangle \\
& =\int_{m_{t h}}^{\infty} \mathrm{dm} d_{S}(m) e^{-i \sqrt{m^{2}+\mathbf{p}^{2}} t}
\end{aligned}
$$

coincides with (1), hence concluding our derivation.

In principle, one could also start from the Hamiltonian $H_{\mathbf{p}}$ and obtain the energy distribution associated with this state, denoted as $d_{S}^{\mathbf{p}}(E)$. Then, $a_{S}^{\mathbf{p}}(t)$ should also emerge as the Fourier transform of the latter. This is hard to do here, since the explicit expression of $H_{\mathbf{p}}$ in terms of $|S, \mathbf{p}\rangle$ and $|\mathbf{k}, \mathbf{p}\rangle$ was not written down (as mentioned previously, this is not an easy task). Quite interestingly, in the framework of QFT, the function $d_{S}^{\mathbf{p}}(E)$ can be easily determined; see Section 3 . 
As a last comment of this section, we recall that the general nondecay probability of an arbitrary state $|\Psi\rangle$ reads

$$
P_{|\Psi\rangle}(t)=\int \mathrm{d}^{3} \mathrm{p}\left|\left\langle S, \mathbf{p}\left|e^{-i H t}\right| \Psi\right\rangle\right|^{2},
$$

whose interpretation is straightforward: we project $|\Psi\rangle$ onto the basis of undecayed states. In general, $P_{|\Psi\rangle}(0)$ is not unity. Notice also that $P_{|\Psi\rangle}(t)$ is not the survival probability of the state $|\Psi\rangle$ (a state can change with time but still be undecayed if it is a different superposition of $|S, \mathbf{p}\rangle)$.

When a boost $U_{\mathbf{v}}$ on the state with zero momentum (and hence with zero velocity) $|S, \mathbf{0}\rangle$ is considered, the resulting state reads [17]

$$
\begin{aligned}
\left|\varphi_{\mathbf{v}}\right\rangle & =U_{\mathbf{v}}|S, \mathbf{0}\rangle \\
& =\int_{m_{t h}}^{\infty} \mathrm{dm} \alpha_{S}(m) \sqrt{m} \gamma^{3 / 2}|m, m \gamma \mathbf{v}\rangle,
\end{aligned}
$$

where $\gamma=\left(1-\mathbf{v}^{2}\right)^{-1 / 2}$. In fact, each element of the superposition, $|m, m \gamma \mathbf{v}\rangle$, has velocity $\mathbf{v}$. Of course, $\left|\varphi_{\mathbf{v}}\right\rangle$ is not an eigenstate of momentum, since each element in (26) has a different momentum $\mathbf{p}=m \gamma \mathbf{v}$. In this respect, the state $|S, \mathbf{0}\rangle$ is special: it is the only state which has at the same time definite momentum and definite velocity (both of them vanishing). As mentioned in the Introduction, the nondecay probability associated with $\left|\varphi_{\mathbf{v}}\right\rangle$ vanishes:

$$
P_{\left|\varphi_{\mathbf{v}}\right\rangle}(t)=0 \quad \forall \mathbf{v} \neq \mathbf{0} .
$$

As soon as a nonzero velocity is considered, the state has decayed. This result is quite surprising but also rather "delicate": when a wave packet is considered, $P_{n d}^{\left|\varphi_{\mathrm{v}}\right\rangle}(t)$ is nonzero (even if it is not 1 for $t=0$ ) [17].

\section{Covariant QFT Derivation of (1)}

Let us consider an unstable particle described by the scalar field $S(x) \equiv S(t, \mathbf{x})$. For an illustrative example, one can couple $S$ with bare mass $M_{0}$ to two scalar fields $\varphi_{1}$ (with mass $m_{1}$ ) and $\varphi_{2}$ (with mass $m_{2}$ ) via the interaction term $g S \varphi_{1} \varphi_{2}$, leading to the QFT Lagrangian

$$
\begin{aligned}
\mathscr{L}= & \frac{1}{2}\left[\left(\partial_{\mu} \varphi_{1}\right)^{2}-m_{1}^{2} \varphi_{1}^{2}\right]+\frac{1}{2}\left[\left(\partial_{\mu} \varphi_{2}\right)^{2}-m_{2}^{2} \varphi_{2}^{2}\right] \\
& +\frac{1}{2}\left[\left(\partial_{\mu} S\right)^{2}-M_{0}^{2} S^{2}\right]+g S \varphi_{1} \varphi_{2} .
\end{aligned}
$$

This is the QFT counterpart of the QM system of the previous section. Note we use scalar fields for simplicity, but our discussion is in no way limited to it.

The (full) propagator of the state $S$ (details in [24]) reads

$$
\Delta_{S}\left(p^{2}\right)=\frac{1}{p^{2}-M_{0}^{2}+\Pi\left(p^{2}\right)+i \varepsilon}
$$

$$
\text { with } p^{2}=E^{2}-\mathbf{p}^{2}
$$

where $E=p^{0}$ is the energy and $\mathbf{p}$ the three-momentum. Because of covariance, $\Delta_{S}\left(p^{2}\right)$ depends only on $p^{2}$. The quantity $\Pi\left(p^{2}\right)$ is the one-particle irreducible diagram. Its calculation is of course nontrivial (it requires a proper regularization), but it is not needed for our purposes. The imaginary part is

$$
\begin{aligned}
\operatorname{Im} \Pi\left(p^{2}\right) & =\sqrt{p^{2}} \Gamma\left(\sqrt{p^{2}}\right) \\
& =\frac{|\mathbf{k}|}{8 \pi \sqrt{p^{2}}} g^{2} f_{\Lambda}^{2}(|\mathbf{k}|)+\cdots,
\end{aligned}
$$

where dots refer to higher orders, which are however typically very small [25]. Once $\operatorname{Im} \Pi\left(p^{2}\right)$ is fixed, $\operatorname{Re} \Pi\left(p^{2}\right)$ can be determined by dispersion relations (for an example of this technique, see, e.g., [26]). The quantity $\Gamma^{t l}=\Gamma\left(\sqrt{p^{2}}=M\right)$ is the usual tree-level decay width; hence in the exponential limit the decay law $P_{S}(t)=e^{-\Gamma^{t l} t}$ must be reobtained. As mentioned in the Introduction, an unstable state has not a definite mass: this is why different definitions for $M$ (which is not the bare mass $M_{0}$ entering in (29)) are possible: $\operatorname{Re} \Delta_{S}^{-1}\left(p^{2}=M^{2}\right)=0$ (zero of the real part of the denominator), or $\operatorname{Re}\left[\sqrt{s_{\text {pole }}}\right]$, with $\Delta_{S}^{-1}\left(s_{\text {pole }}\right)=0$ (real part of the pole), or the maximum of the spectral function defined below.

We also recall that

$$
|\mathbf{k}|=\sqrt{\frac{p^{4}+\left(m_{1}^{2}-m_{2}^{2}\right)^{2}-2 p^{2}\left(m_{1}^{2}+m_{2}^{2}\right)}{4 p^{2}}}
$$

coincides, for the on-shell decay, with the modulus of the three-momentum of one of the outgoing particles. The vertex function $f_{\Lambda}(|\mathbf{k}|)$ is a proper regularization which fulfills the condition $f_{\Lambda}(|\mathbf{k}| \rightarrow 0)=1$ and describes the highenergy behavior of the theory (its UV completion); hence the parameter $\Lambda$ is some high-energy scale; $f_{\Lambda}(|\mathbf{k}|)$ is formally not present in (28) since it appears in the regularization procedure, but it can be included directly in the Lagrangian by rendering it nonlocal [27] in a way that fulfills covariance [28]. In a renormalizable theory (such as the one of (28)), the dependence on $\Lambda$ disappears in the low-energy limit.

The properties outlined above, although in general very important in specific calculations, turn out to be actually secondary to the proof that we present below, where only the formal expression of the propagator of (29) is relevant. Moreover, even when the unstable particle is not a scalar, one can always define a scalar part of the propagator which looks just as in (29), then the outlined properties apply, mutatis mutandis, to each QFT Lagrangian.

As a next step, upon introducing the Mandelstam variable $s=p^{2}$, the function $F(s)$ defined as

$$
F(s)=\frac{1}{\pi} \operatorname{Im}\left[\Delta_{S}\left(p^{2}=s\right)\right]
$$

fulfills the normalization condition:

$$
\int_{s_{t h}}^{\infty} \mathrm{d} F(s)=1
$$


where $s_{t h}=m_{t h}^{2}$ is the minimal squared energy. For the case of (28), one has obviously $s_{t h}=\left(m_{1}+m_{2}\right)^{2}$. The normalization (33) is a consequence of the Källén-Lehmann representation [29]

$$
\Delta_{S}\left(p^{2}\right)=\int_{s_{t h}}^{\infty} \mathrm{ds} \frac{F(s)}{p^{2}-s+i \varepsilon}
$$

in which the propagator $\Delta_{S}\left(p^{2}\right)$ has been rewritten as the "sum" of free propagators $\left[p^{2}-s+i \varepsilon\right]^{-1}$, each one of them weighted by $F(s)$ : $\operatorname{ds} F(s)$ is the probability that the squared mass lies between $s$ and $s+$ ds. Of course, the normalization (33) is a very important feature of our approach. For a detailed proof of its validity, we refer to [30]. Here we recall a simple version of it, which is obtained by assuming the rather strong requirement $\Pi\left(p^{2}\right)=0$ for $p^{2}>\Lambda^{2}$, where $\Lambda$ is a high-energy scale (no matter how large). Under this assumption

$$
\begin{aligned}
\Delta_{S}\left(p^{2}\right) & =\frac{1}{p^{2}-M_{0}^{2}+\Pi\left(p^{2}\right)+i \varepsilon} \\
& =\int_{s_{t h}}^{\Lambda^{2}} \mathrm{ds} \frac{F(s)}{p^{2}-s+i \varepsilon} .
\end{aligned}
$$

Then, upon taking a certain value $p^{2} \gg \Lambda^{2}$, the previous equation reduces to

$$
\begin{aligned}
\frac{1}{p^{2}} & =\int_{s_{t h}}^{\Lambda^{2}} \mathrm{~d} s \frac{F(s)}{p^{2}} \longrightarrow \\
\int_{s_{t h}}^{\Lambda^{2}} \mathrm{~d} s F(s) & =1
\end{aligned}
$$

The general case in which $\Pi\left(p^{2} \rightarrow \infty\right)=0$ smoothly requires more steps, but the final result of (33) still holds [30].

Let us now consider the rest frame of the decaying particle: $\mathbf{p}=\mathbf{0}, s=p^{2}=E^{2}=m^{2}$. Here, upon a simple variable change $(m=\sqrt{s})$, we obtain the mass distribution (or spectral function) $d_{S}^{\mathrm{p}=\mathbf{0}}(m)$ through the equation

$$
\mathrm{dm} d_{S}^{\mathbf{p}=\mathbf{0}}(m)=\mathrm{ds} F(s),
$$

out of which

$$
d_{S}(m)=d_{S}^{\mathbf{p}=\mathbf{0}}(m)=2 m F\left(s=m^{2}\right) .
$$

As already mentioned, $\operatorname{dm} d_{S}(m)$ is the probability that the particle $S$ has a mass between $m$ and $m+\operatorname{dm}[24,31]$. In this context, the normalization

$$
\int_{m_{t h}}^{\infty} \mathrm{dm} d_{S}(m)=1
$$

follows from (33). Once the function $d_{S}(m)$ is identified as the mass distribution of the undecayed quantum state, the nondecay probability's amplitude $a_{S}^{0}(t)$ can be obtained by repeating the steps of Section 2 . The result coincides, as expected, with (3). Yet, it should be stressed that the unstable quantum state $|S, \mathbf{0}\rangle$ characterized by the distribution $d_{S}(m)$ is not simply given by $a_{0}^{\dagger}\left|0_{P T}\right\rangle$, where $\left|0_{P T}\right\rangle$ is the perturbative vacuum and $a_{\mathbf{p}}^{\dagger}$ the creator operator of the noninteracting field $S$. The case of neutrino oscillations shows a similar situation: the state corresponding to a certain flavour, such as the neutrino $v_{e}$, must be constructed with due care by making use of Bogolyubov transformations [32]. Along this line, the exact and formal determination of the state $|S, \mathbf{0}\rangle$, corresponding to the mass distribution $d_{S}(m)$, in the context of QFT requires a generalization of Bogolyubov transformations and was, to our knowledge, not yet explicitly done (it is left for the future). Nevertheless, it is not needed for the purpose of this paper.

Let us now consider the particle $S$ moving with a certain momentum p. Upon using $s=E^{2}-\mathbf{p}^{2}$, the energy distribution-as function of $E$-is obtained by

$$
\mathrm{dE} d_{S}^{\mathbf{p}}(E)=\mathrm{ds} F(s),
$$

leading to

$$
\begin{aligned}
d_{S}^{\mathbf{p}}(E) & =2 E F\left(s=E^{2}-\mathbf{p}^{2}\right) \\
& =\frac{E}{\sqrt{E^{2}-\mathbf{p}^{2}}} d_{S}\left(\sqrt{E^{2}-\mathbf{p}^{2}}\right) .
\end{aligned}
$$

The quantity $\mathrm{dE} d_{S}^{\mathbf{p}}(E)$ is the probability that the particle $S$ with definite momentum $\mathbf{p}$ has an energy between $E$ and $E+\mathrm{dE}\left(\right.$ clearly, $\left.d_{S}^{\mathbf{p}=\mathbf{0}}(E)=d_{S}(m=E)\right)$. Also in this case, the normalization

$$
\int_{\sqrt{m_{t h}^{2}+\mathbf{p}^{2}}}^{\infty} \mathrm{dE} d_{S}^{\mathbf{p}}(E)=1
$$

is a consequence of (33). When $d_{S}(m)$ has a maximum at $M$, then $d_{S}^{\mathbf{p}}(E)$ has a maximum at $\sim \sqrt{M^{2}+\mathbf{p}^{2}}$. Note the very fact that the propagator depends on $p^{2}=E^{2}-\mathbf{p}^{2}$ allows to determine the spectral function $d_{S}^{\mathrm{p}}(E)$ for a definite momentum $\mathbf{p}$, that corresponds to the state $|S, \mathbf{p}\rangle$ of Section 2.

The nondecay probability's amplitude for a state $S$ moving with momentum $\mathbf{p}$ is then given by

$$
a_{S}^{\mathbf{p}}(t)=\int_{\sqrt{m_{t h}^{2}+\mathbf{p}^{2}}}^{\infty} \mathrm{dE} d_{S}^{\mathbf{p}}(E) e^{-i E t},
$$

where we have taken into account that the minimal energy is now given by $\sqrt{m_{t h}^{2}+\mathbf{p}^{2}}$.

This expression can be manipulated by using (41) and via a change of variable:

$$
\begin{aligned}
a_{S}^{\mathbf{p}}(t) & =\int_{\sqrt{m_{t h}^{2}+\mathbf{p}^{2}}}^{\infty} \mathrm{dE} d_{S}^{\mathbf{p}}(E) e^{-i E t} \\
& =\int_{\sqrt{m_{t h}^{2}+\mathbf{p}^{2}}}^{\infty} \mathrm{dE} \frac{E}{\sqrt{E^{2}-\mathbf{p}^{2}}} d_{S}\left(\sqrt{E^{2}-\mathbf{p}^{2}}\right) e^{-i E t} \\
& =\int_{m_{t h}}^{\infty} \mathrm{dm} d_{S}(m) e^{-i \sqrt{m^{2}+\mathbf{p}^{2}} t},
\end{aligned}
$$

which coincides exactly with (1), as we wanted to demonstrate. Thus, we confirm the validity of (1) in a covariant QFTbased framework. 


\section{Conclusions}

The decay law of a moving unstable particle is an interesting subject that connects special relativity to QM and QFT. An important aspect is the validity of (1), which expresses the nondecay probability of a state with nonzero momentum and whose standard derivation is reviewed in Section 2.

The main contribution of this paper has been the derivation of a quantum field theoretical proof of (1). To this end, we started from the (scalar part of the) propagator of an unstable quantum field, denoted as $S$. Then, we have determined the energy distribution of the state $S$ with definite momentum $\mathbf{p}$, out of which the survival's probability amplitude is calculated.

As discussed in the Introduction, there are interesting and peculiar consequences of (1). Future studies are definitely needed to further understand the properties of a decay of a moving unstable particle and to look for feasible experimental tests.

\section{Data Availability}

There is no external data. Theoretical result is based on the author's research.

\section{Conflicts of Interest}

The author declares that there are no conflicts of interest regarding the publication of this paper.

\section{Acknowledgments}

The author thanks S. Mrówczyński and G. Pagliara for useful discussions.

\section{References}

[1] L. A. Khalfin, "Zhurnal Eksperimentalnoi i Teoreticheskoi Fiziki," Journal for Experimental and Theoretical Physics, vol. 6, p. 1053, 1371, (Engl. trans. Sov. Phys. JETP 6 1053).

[2] B. Misra and E. C. Sudarshan, "The Zeno's paradox in quantum theory," Journal of Mathematical Physics, vol. 18, no. 4, pp. 756763, 1977.

[3] A. Degasperis, L. Fonda, and G. C. Ghirardi, "Does the Lifetime of an Unstable System Depend on the Measuring Apparatus?" Nuovo Cim, vol. 21, no. 3, pp. 471-484, 1973.

[4] L. Fonda, G. C. Ghirardi, and A. Rimini, "Decay theory of unstable quantum systems," Reports on Progress in Physics, vol. 41, no. 4, p. 587, 1978.

[5] P. Facchi, H. Nakazato, and S. Pascazio, "From the Quantum Zeno to the Inverse Quantum Zeno Effect," Physical Review Letters, vol. 86, no. 13, pp. 2699-2703, 2001.

[6] P. Facchi and S. Pascazio, "Spontaneous emission and lifetime modification caused by an intense electromagnetic field," Physical Review A, vol. 62, article 023804, 2000.

[7] K. Urbanowski and K. Raczyńska, "Possible emission of cosmic $X$ - and $\gamma$-rays by unstable particles at late times," Physics Letters $B$, vol. 731, pp. 236-241, 2014.

[8] M. Peshkin, A. Volya, and V. Zelevinsky, "Non-exponential and oscillatory decays in quantum mechanics," EPL (Europhysics Letters), vol. 107, no. 4, p. 40001, 2014.
[9] F. Giacosa, "Non-exponential decay in quantum field theory and in quantum mechanics: the case of two (or more) decay channels," Foundations of Physics, vol. 42, no. 10, pp. 1262-1299, 2012.

[10] S. R. Wilkinson, C. F. Bharucha, M. C. Fischer et al., "Experimental evidence for non-exponential decay in quantum tunnelling," Nature, vol. 387, no. 6633, pp. 575-577, 1997.

[11] C. Rothe, S. I. Hintschich, and A. P. Monkman, "Violation of the Exponential-Decay Law at Long Times," Physical Review Letters, vol. 96, no. 16, 2006.

[12] F. Giacosa and G. Pagliara, "Deviation from the exponential decay law in relativistic quantum field theory: the example of strongly decaying particles," Modern Physics Letters A, vol. 26, no. 30, pp. 2247-2259, 2011.

[13] L. A. Khalfin, "Quantum Theory of unstable particles and relativity," PDMI Pewprint, 1997.

[14] M. I. Shirokov, "Decay law of moving unstable particle," International Journal of Theoretical Physics, vol. 43, no. 6, pp. 1541-1553, 2004.

[15] E. V. Stefanovich, "Quantum effects in relativistic decays," International Journal of Theoretical Physics, vol. 35, no. 12, pp. 2539-2554, 1996.

[16] K. Urbanowski, "Decay law of relativistic particles: quantum theory meets special relativity," Physics Letters B, vol. 737, pp. 346-351, 2014.

[17] F. Giacosa, "Decay law and time dilatation," Acta Physica Polonica B, vol. 47, no. 9, pp. 2135-2150, 2016.

[18] V. Weisskopf, E. P. Wigner, and Z. Phys, "Berechnung der natürlichen Linienbreite auf Grund der Diracschen Lichttheorie," Zeitschrift für Physik, vol. 63, no. 1-2, pp. 54-73, 1930.

[19] M. O. Scully and M. S. Zubairy, Quantum Optics, Cambridge University Press, Cambridge, UK, 1997.

[20] J. Bailey et al., "Measurements of relativistic time dilatation for positive and negative muons in a circular orbit," Nature, vol. 26, pp. 301-305, 1977.

[21] S. A. Alavi and C. Giunti, "Which is the quantum decay law of relativistic particles?” EPL (Europhysics Letters), vol. 109, no. 6, 2015.

[22] T. D. Lee, "Some Special Examples in Renormalizable Field TheoryT. D. Lee," Physical Review, vol. 95, no. 5, p. 1329, 1954.

[23] Z. Liu, W. Kamleh, D. B. Leinweber, F. M. Stokes, A. W. Thomas, and J. Wu, "Hamiltonian Effective Field Theory Study of the," Physical Review Letters, vol. 116, no. 8, 2016.

[24] F. Giacosa and G. Pagliara, "Spectral functions of scalar mesons," Physical Review C: Nuclear Physics, vol. 76, no. 6, 2007.

[25] J. Schneitzer, T. Wolkanowski, and F. Giacosa, "The role of the next-to-leading order triangle-shaped diagram in two-body hadronic decays," Nuclear Physics. B. Theoretical, Phenomenological, and Experimental High Energy Physics. Quantum Field Theory and Statistical Systems, vol. 888, pp. 287-299, 2014.

[26] T. Wolkanowski, F. Giacosa, and D. H. Rischke, Physical Review D: Particles, Fields, Gravitation and Cosmology, vol. 93, no. 1, 2016.

[27] J. Terning, “Gauging nonlocal Lagrangians," Physical Review D, vol. 44, p. 887, 1991.

[28] M. Soltysiak and F. Giacosa, "A covariant nonlocal Lagrangian for the description of the scalar kaonic sector," Acta Physica Polonica B, 9, 2016, https://arxiv.org/abs/1607.01593.

[29] M. E. Peskin and D. V. Schroeder, An Introduction to Quantum Field Theory, Addison-Wesley, Reading, Mass, USA, 1995. 
[30] F. Giacosa and G. Pagliara, "Spectral function of a scalar boson coupled to fermions," Physical Review D: Particles, Fields, Gravitation and Cosmology, vol. 88, no. 2, 2013.

[31] P. T. Matthews and A. Salam, "Relativistic theory of unstable particles," Physical Review, vol. 112, p. 283, 1958.

[32] M. Blasone, A. Capolupo, O. Romei, and G. Vitiello, "Quantum field theory of boson mixing," Physical Review D: Particles, Fields, Gravitation and Cosmology, vol. 63, no. 12, Article ID 125015, 2001. 

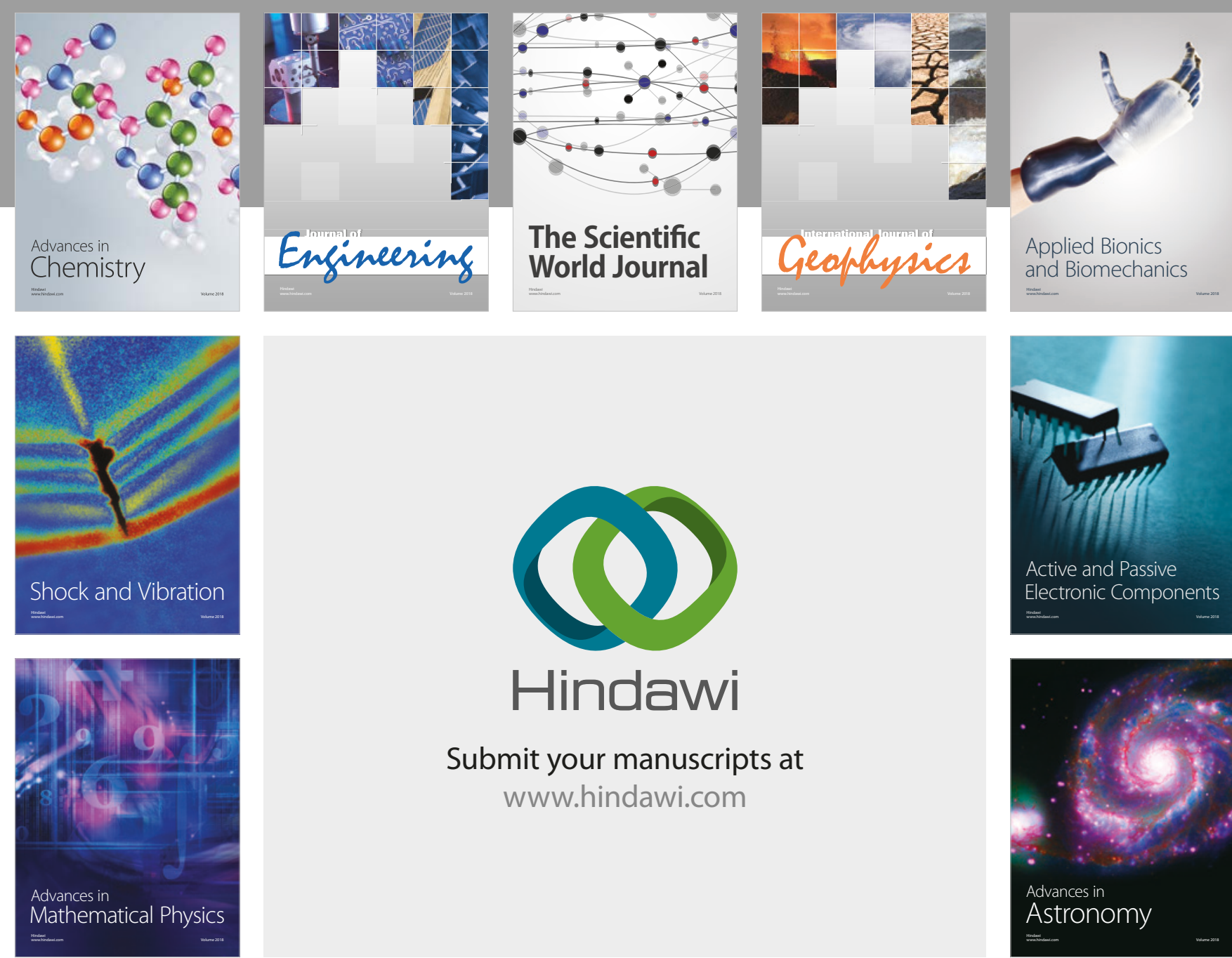

Submit your manuscripts at

www.hindawi.com

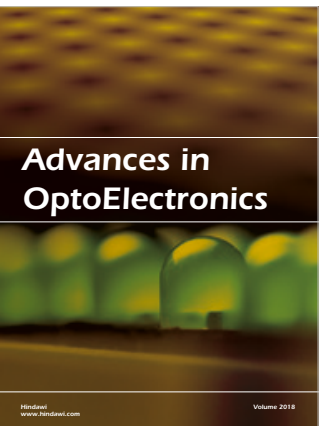

\section{Rotcting Machinery}
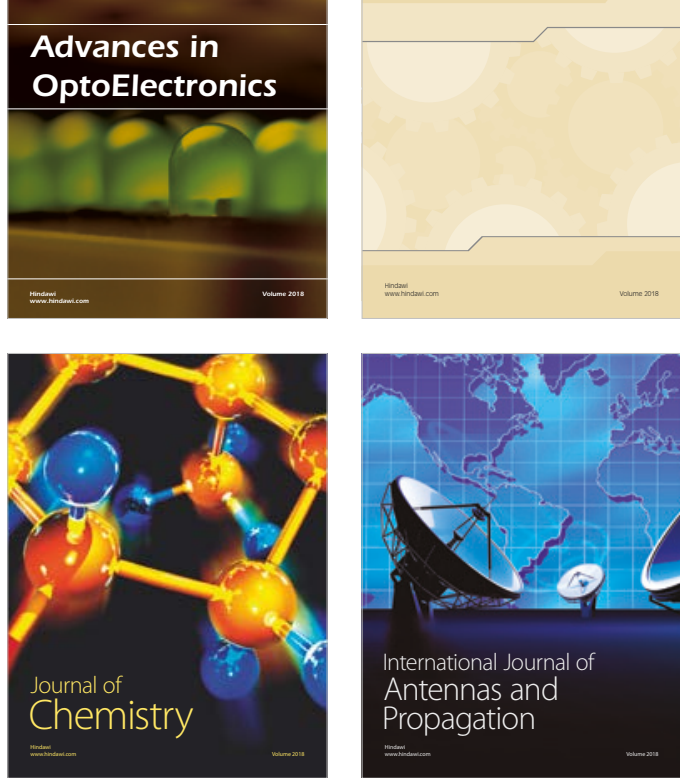

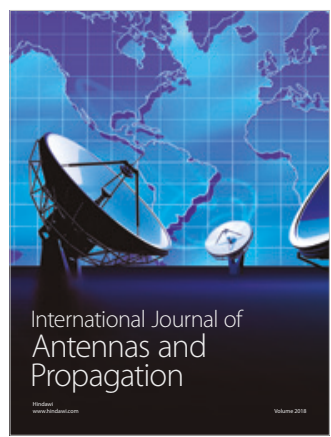

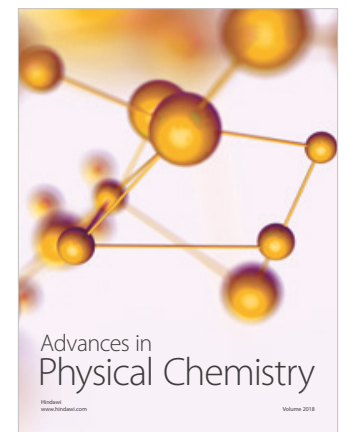

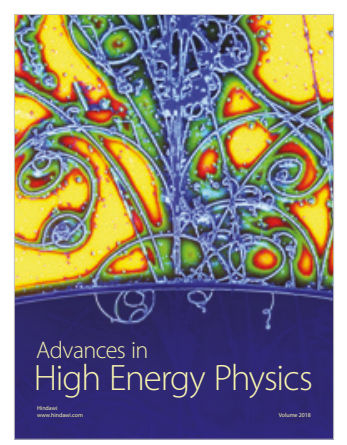

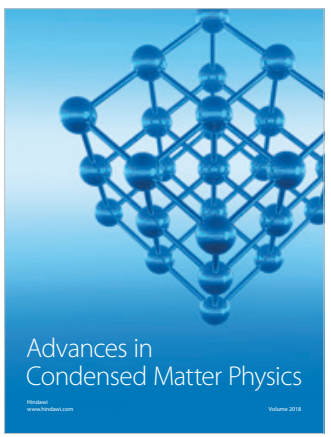

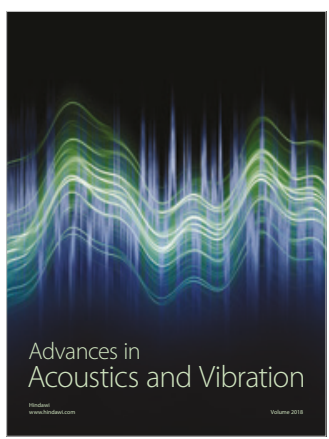

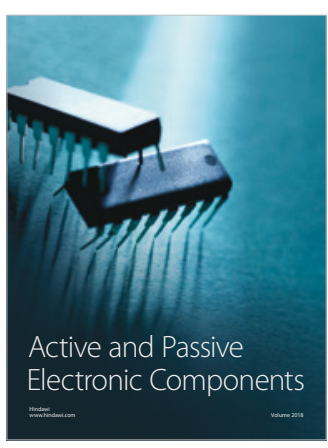
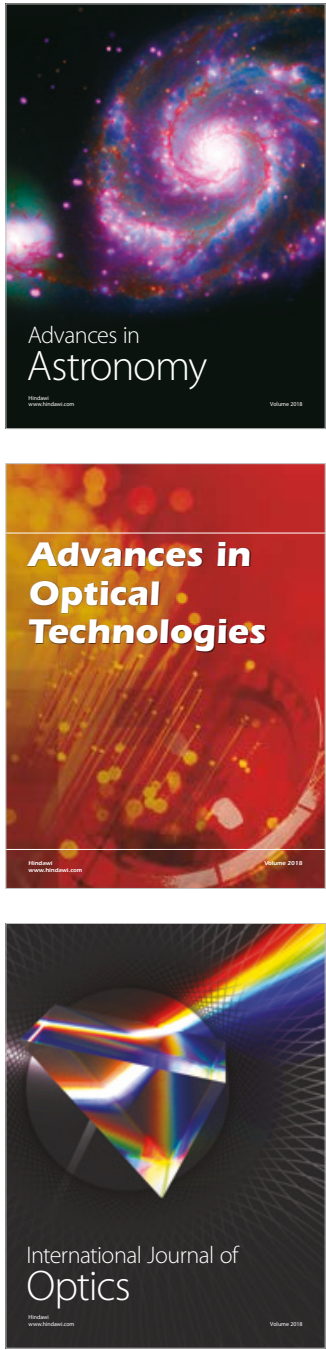